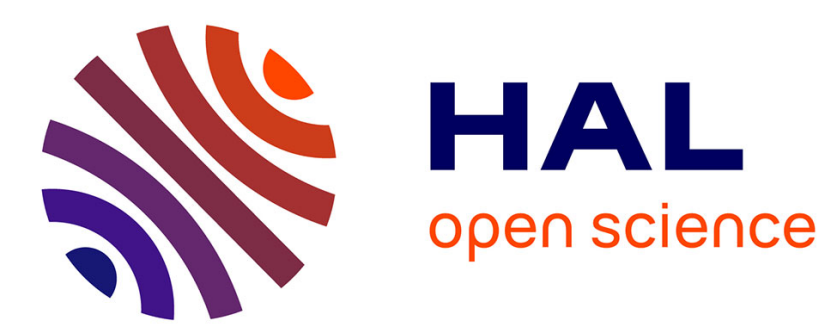

\title{
Sensitivity of SHG-FROG for the Characterisation of Ultrahigh-Repetition-Rate Telecommunication Laser Sources
}

\author{
Julien Fatome, Stéphane Pitois, Guy Millot
}

\section{To cite this version:}

Julien Fatome, Stéphane Pitois, Guy Millot. Sensitivity of SHG-FROG for the Characterisation of Ultrahigh-Repetition-Rate Telecommunication Laser Sources. Optical Fiber Technology, 2004, 10 (1), pp.73-78. 10.1016/j.yofte.2003.08.001 . hal-00475077

\section{HAL Id: hal-00475077 \\ https://hal.science/hal-00475077}

Submitted on 21 Apr 2010

HAL is a multi-disciplinary open access archive for the deposit and dissemination of scientific research documents, whether they are published or not. The documents may come from teaching and research institutions in France or abroad, or from public or private research centers.
L'archive ouverte pluridisciplinaire HAL, est destinée au dépôt et à la diffusion de documents scientifiques de niveau recherche, publiés ou non, émanant des établissements d'enseignement et de recherche français ou étrangers, des laboratoires publics ou privés. 


\title{
Sensitivity of SHG-FROG for the Characterization of Ultrahigh-Repetition-Rate Telecommunication Laser Sources
}

\author{
Julien Fatome, Stéphane Pitois, and Guy Millot ${ }^{1}$ \\ Laboratoire de Physique de l'Université de Bourgogne, \\ Unité Mixte de Recherche CNRS 5027 \\ Faculté des Sciences Mirande, 9 Avenue A. Savary, B.P. 47 870, 21078 Dijon, France
}

\begin{abstract}
We analyze experimentally the sensitivity of second-harmonic generation frequencyresolved optical gating (SHG-FROG) for the complete intensity and phase characterization of both a sinusoidal beat signal and a train of $1.3 \mathrm{ps}$ pulses at a repetition rate of $160 \mathrm{GHz}$ at $1550 \mathrm{~nm}$. Using a commercially-available optical spectrum analyzer in the SHG-FROG setup, incident pulses with energies of only $125 \mathrm{fJ}$ and $190 \mathrm{fJ}$, which correspond to the beat signal and the $1.3 \mathrm{ps}$ pulse train, respectively, have been accurately characterized.
\end{abstract}

Running head: SHG-FROG FOR ULTRAFAST TELECOMMUNICATIONS

${ }^{1}$ To whom correspondence should be addressed. E-mail: Guy.Millot@ u-bourgogne.fr. 


\section{INTRODUCTION}

In ultrafast telecommunication systems using optical time-division multiplexing, picosecond and subpicosecond optical pulses will be transmitted through optical fibres at repetition rates of several hundreds of Gbit/s [1]. However, chromatic dispersion and Kerr effects may cause severe distortions in the shape and chirp of the transmitted ultrashort pulses. Thus, the ability to measure the fundamental parameters of optical pulses as well as pulse distortions after propagation in a high-speed transmission line is of major importance for the optimization of input laser sources and the design of high-speed transmission lines.

In this context, the ultrashort pulse measurement technique of frequency resolved optical gating (FROG) is now a well-established method to retrieve intensity and phase of ultrashort pulses [2] and has been successfully applied in the third telecommunication window around $1550 \mathrm{~nm}$, to characterize isolated single pulses from sources having repetition rates ranging from $4.2 \mathrm{MHz}$ to $40 \mathrm{GHz}$ [3-7]. In addition, FROG has been extended to allow the characterization of periodic pulse trains at ultrahigh repetition rates ranging from several hundreds of $\mathrm{GHz}$ to a few $\mathrm{THz}$, although this work has, to date, been restricted to pulse trains in the visible with peak powers in the $100 \mathrm{~W}$ range [8]. Moreover, it has recently been shown that FROG inversion algorithms can accurately retrieve relatively elaborated optical fields such as that of a pseudo-random sequence of chirped pulses [9]. The FROG technique appears therefore as an essential tool for analysis of ultrahigh bit rates generation and transmission in modern telecommunication experiments.

However, to the best of our knowledge, FROG has not yet been applied to the characterization of pulse trains with ultrahigh repetition rates $(>40 \mathrm{GHz})$ at telecommunication wavelengths. In this paper, we report the experimental characterization 
of $160 \mathrm{GHz}$ pulse trains around $1550 \mathrm{~nm}$ with a FROG system based on second-harmonic generation (SHG) in a BBO crystal and a commercial optical spectrum analyzer. In particular, we study experimentally the sensitivity of SHG-FROG for the characterization of both a beat signal and a 1.3ps pulse train. An important feature of our results when compared with single pulse characterization [3-7] is that the accurate characterization of ultrashort pulse trains allows the recovery of the phase jump between adjacent pulses.

\section{SHG-FROG SETUP}

Figure $1 a$ shows the SHG-FROG setup based on the spectral analysis of the SHG autocorrelation signal using an optical spectrum analyzer (OSA, Anritsu MS9710B). The two time-delayed replicas of the input signal were focused (in a background-free geometry) in a $2 \mathrm{~mm}$-thick BBO crystal by a $5 \mathrm{~cm}$ focusing lens. The bandwidth of the phase-matching efficiency for the crystal was estimated to be larger than $100 \mathrm{~nm}$. A variable time delay between the two replicas was tuned by means of a step-motor controlled translation stage. A polarization controller was used to minimize the insertion loss of the polariser placed at the autocorrelator input and to optimise the SHG signal. The FROG trace was conveniently built up by adjusting the autocorrelator to a particular delay, and then by scanning the OSA wavelength with a spectral resolution of $0.07 \mathrm{~nm}$. The measured spectra were then interpolated onto a $256 \times 256$ grid, and used as input to the algorithm for the retrieval of the periodic pulse-train characteristics [8].

At this point, we discuss several features related to the definition of the sensitivity of an SHG-FROG set-up. First let us recall that the sensivity of an autocorrelator can be 
defined as the product of the peak and average powers of the incident pulse which is characterized $\left(\mathrm{P}_{\text {average }} \times \mathrm{P}_{\text {peak }}\right)$. For an autocorrelator, this measure of sensitivity is widelyused because it is directly proportional to the integrated average power of the second harmonic autocorrelation signal at zero delay. However, in the case of an SHG-FROG setup, this measure is inappropriate because the SHG signal is spectrally resolved. As a result, a correct definition of the sensitivity of an SHG-FROG signal is significantly more complicated, as it must take into account the different types of incident field and the particular type of spectral analysis which is being carried out, either using a fixed grating and CCD array, or a rotating grating and a fixed detector, but this is outside the scope of this paper.

\section{EXPERIMENTAL RESULTS}

\subsection{0-GHz Sinusoidal Beat Signal}

The first experiment that we carried out is the characterization of a sinusoidal beat signal at $160 \mathrm{GHz}$, with well-known intensity and phase, obtained from the superposition of two continuous waves centred around 1550nm. Both waves were delivered by tunable external cavity lasers (ECL's) subsequently amplified by an Erbium amplifier, as shown in Fig. $1 b$. The two wave powers were set to the same value and the polarization states were aligned parallel. Figures $2 a$ and $b$ show the measured and the retrieved FROG traces of this beat signal, respectively. The total average power was fixed to $13 \mathrm{dBm}$ at the input of the autocorrelator, which corresponds to a pulse (cycle) energy of 125fJ. The retrieved intensity and phase, shown in Fig. $2 c$, exhibit the expected characteristics of a $100 \%$ 
modulated sinusoidal beat signal. The retrieval error was found to be $G=0.011$. As can be seen in Figs. $2 d$ and $e$, respectively, the excellent agreement between the experimental spectrum and autocorrelation function of the beat signal directly measured (solid line), and those calculated from the retrieved field (circles), confirms the reliability of our characterization system.

\subsection{0-GHz Train of 1.3-ps Pulses}

Another series of experiments was carried out in order to characterize a 160-GHz train of 1.3-ps pulses. This pulse train was generated using multiple four-wave mixing temporal compression of a beat signal in the anomalous-dispersion regime $[10,11]$ of a $1 \mathrm{~km}$-long non-zero dispersion-shifted fibre (NZ-DSF). The beat-signal average power was set to 27.2dBm. Stimulated Brillouin scattering in the NZ-DSF was suppressed by externally modulating the two ECL's with a phase modulator (see Fig. 1b). The NZ-DSF has $0.21 \mathrm{~dB} / \mathrm{km}$ loss, an anomalous dispersion of $1 \mathrm{ps} / \mathrm{nm} / \mathrm{km}$, a nonlinear coefficient of $1.7 \mathrm{~W}^{-}$ ${ }^{1} \mathrm{~km}^{-1}$, and a third-order dispersion of $0.07 \mathrm{ps} / \mathrm{nm}^{2} / \mathrm{km}$. Figure 3 shows the results obtained when the average power of the compressed pulse train was fixed to $23 \mathrm{dBm}$ at the input of the FROG setup. The retrieval error was $G=0.0019$. Let us remark that the intensity and phase profiles show compressed pulses without pedestal or chirp. Figure $3 c$ (crosses) shows the retrieved intensity and phase obtained when the average power measured at the input of the FROG setup was decreased to $14.8 \mathrm{dBm}$, corresponding to a pulse energy of $190 \mathrm{fJ}$, and a peak power of $140 \mathrm{~mW}$. The comparison between the $14.8 \mathrm{dBm}$ (crosses) and the $23 \mathrm{dBm}$ (solid line) results clearly shows that the shape and phase of the compressed 
pulses remain correctly retrieved at the lower power as confirmed by the corresponding small retrieval error $\mathrm{G}=0.007$.

\section{CONCLUSION}

In conclusion, we have shown that the SHG-FROG technique, employing commercially available BBO crystal and OSA, can accurately characterize 1550nm-pulse trains at a repetition rate of $160 \mathrm{GHz}$ with pulse energy (average power) less than 200fJ $(15 \mathrm{dBm})$. Our results represent the first experimental demonstration of the ability of SHGFROG to characterize telecommunication laser sources operating at ultrahigh repetition rates $(>40 \mathrm{GHz})$ and low average power.

\section{ACKNOWLEDGMENTS}

The authors would like to thank Alcatel France for providing them the $1 \mathrm{~km}-\mathrm{NZ}-\mathrm{DSF}$ and H. Bissesur, P. Brindel, J.M. Dudley, P. Grelu, F. Gutty, and P. Tchofo-Dinda for fruitful discussions. This research was supported by the Conseil régional de Bourgogne, the Centre national de la recherche scientifique (CNRS), and the Ministère de la recherche under contract ACI Jeunes 2015. G. M. acknowledges financial support from the Institut Universitaire de France. 


\section{REFERENCES}

[1] M. Nakazawa, T. Yamamoto, K. Tamura, $1.28 \mathrm{Tbit} / \mathrm{s}-70 \mathrm{~km}$ OTDM transmission using third- and fourth-order simultaneous dispersion compensation with a phase modulator, Electron. Lett. 36 (24) (2000) 2027-2029.

[2] R. Trebino, K.W. Delong, D.N. Fittinghoff, J.N. Sweetser, M.A. Krumbugel, B.A. Richman, D.J. Kane, Measuring ultrashort laser pulses in the time-frequency domain using frequency-resolved optical gating, Rev. Sci. Instrum. 68 (9) (1997) 3277-3295.

[3] F.G. Omenetto, J.W. Nicholson, A.J. Taylor, Second-harmonic generation-frequencyresolved optical gating analysis of low-intensity shaped femtosecond pulses at $1.55 \mu \mathrm{m}$, Opt. Lett. 24 (23) (1999) 1780-1782.

[4] K. Taira, K. Kikuchi, Highly sensitive frequency-resolved optical gating in $1.55 \mu \mathrm{m}$ region using organic nonlinear optical crystal for second-harmonic generation, Electron. Lett. 36 (20) (2000) 1719-1720.

[5] M.D. Thomson, J.M. Dudley, L.P. Barry, J.D. Harvey, Complete pulse characterization at $1.5 \mu \mathrm{m}$ by cross-phase modulation in optical fibers, Opt. Lett. 23 (20) (1998) 1582-1584.

[6] P.A. Lacourt, J.M. Dudley, J.M. Merolla, H. Porte, J.P. Goedgebuer, W.T. Rhodes, Milliwatt peak power pulse characterization at $1.55 \mu \mathrm{m}$ via wavelength conversion frequency resolved optical gating, Opt. Lett. 27 (11) (2002) 863-865.

[7] L.P. Barry, S. Del burgo, B. Thomsen, R.T. Watts, D.A. Reid, J. Harvey, Optimization of optical data transmitters for 40-Gb/s lightwave systems using frequency resolved optical gating, IEEE. Photon. Technol. Lett. 14 (7) (2002) 971-973. 
[8] J.M. Dudley, M.D. Thomson, F. Gutty, S. Pitois, P. Grelu, G. Millot, Complete intensity and phase characterisation of optical pulse trains at terahertz repetition rates, Electron. Lett. 35 (23) (1999) 2042-2044.

[9] P. Grelu, F. Gutty, G. Millot, Pseudo-random pulse sequence characterization with frequency-resolved optical gating, IEEE. Photon. Technol. Lett. 14 (5) (2002) 672-674.

[10] S. Trillo, S. Wabnitz, T.A.B. Kennedy, Non linear dynamics of dual-frequency-pumped multiwave mixing in optical fibers, Phys. Rev. A. 50 (2) (1994) 1732-1747.

[11] S. Pitois, J. Fatome, G. Millot, Generation of 160-GHz transform-limited pedestal-free pulse train through multiwave mixing compression of a dual frequency beat signal, Opt. Lett. 27 (19) (2002) 1729-1731. 


\section{FIGURES CAPTIONS}

Fig. 1 Experimental setup: (a) SHG-FROG system, (b) Pulse train generation at 160GHz.

Fig. 2 Results for sinusoidal beat signal: (a) Experimental SHG-FROG trace, (b) Retrieved SHG-FROG trace, (c) Retrieved intensity (left axis) and phase (right axis), (d) Autocorrelation functions, (e) Spectra calculated from the retrieved intensity and phase “o”, and measured "___ ".

Fig. 3 As in Fig. 2 but for compressed pulse train. 
BS: Beam Splitter

CC: Corner Cube

ML: Micro Lens

P: Polarizer

PC: Polarization Controller

TS: Translation Stage
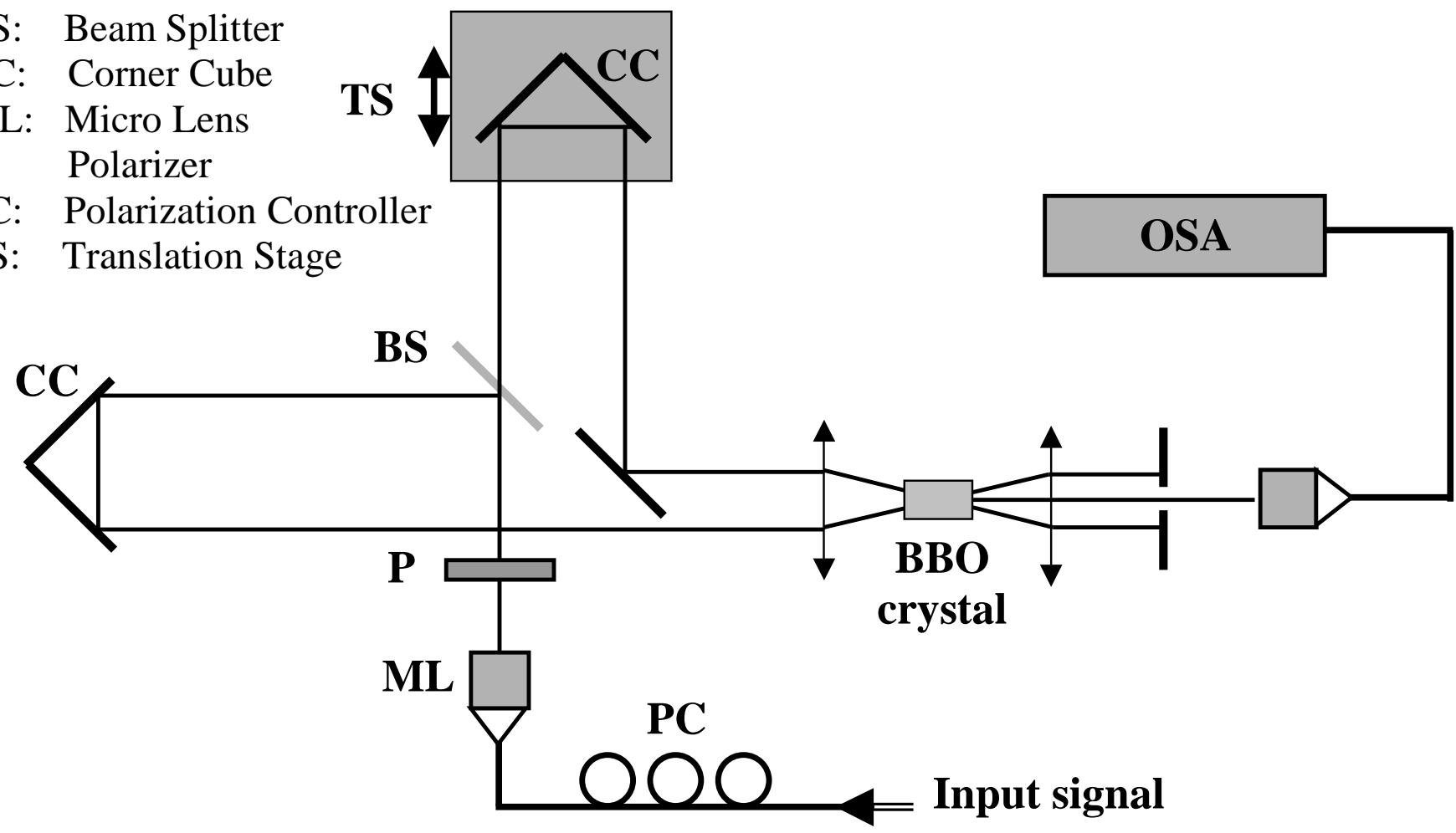

(b)

Tunable CW 50:50

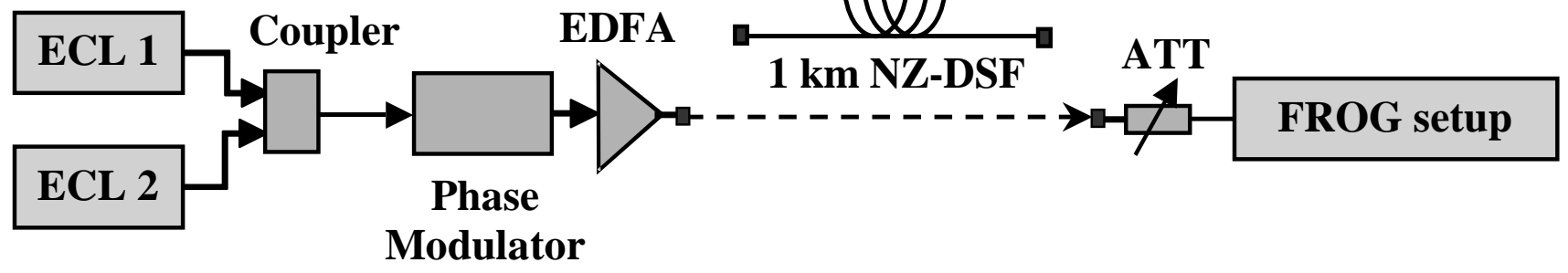


Fatome, Pitois, Millot

(a)

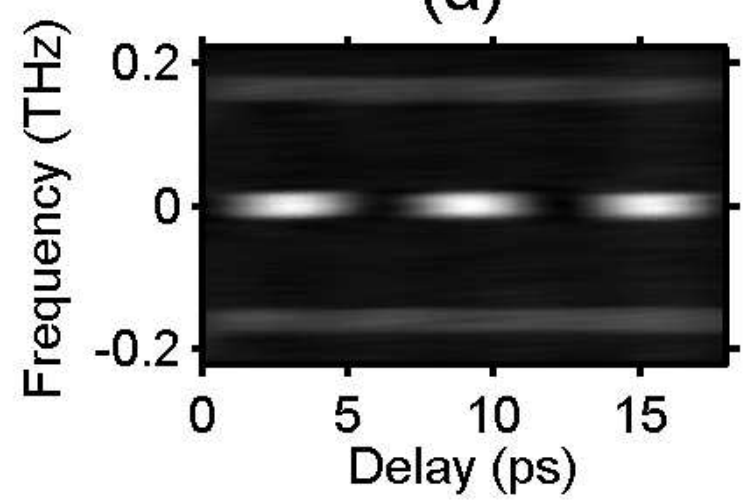

(c)

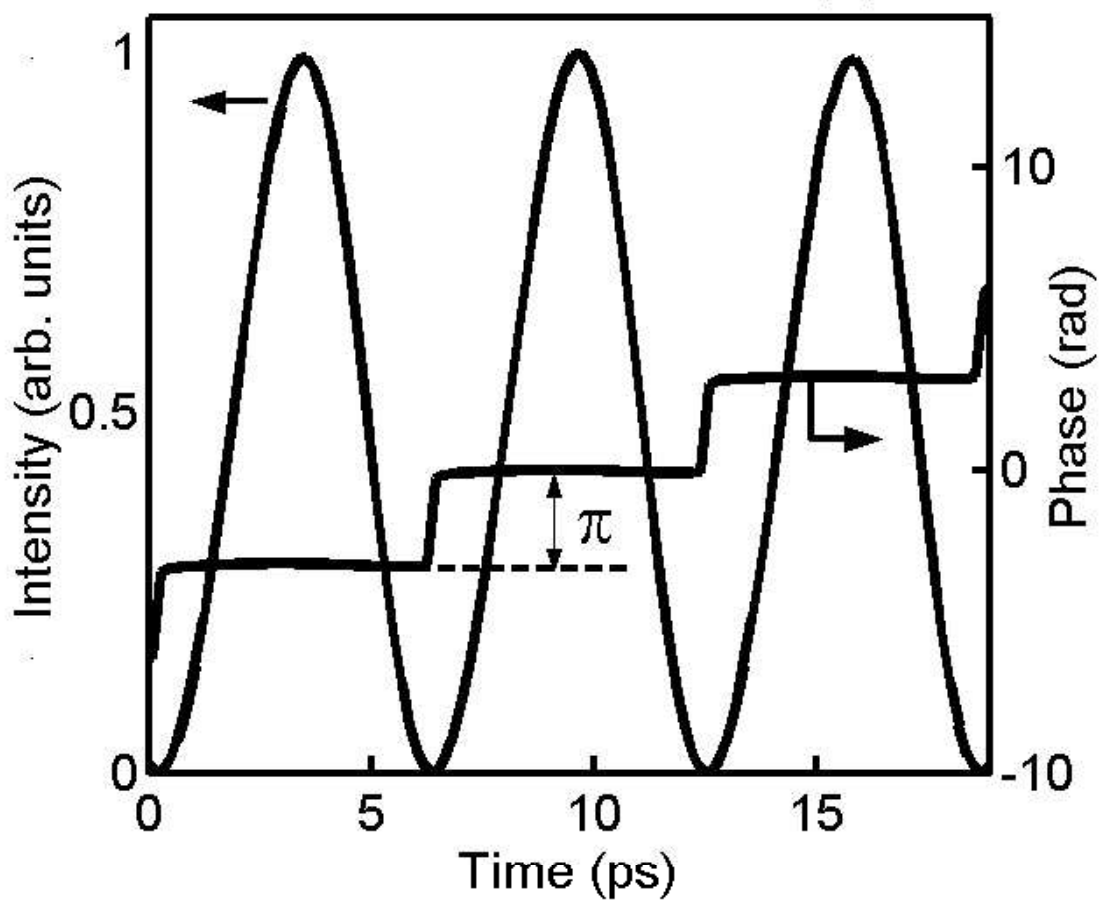

Fig. 2

(b)

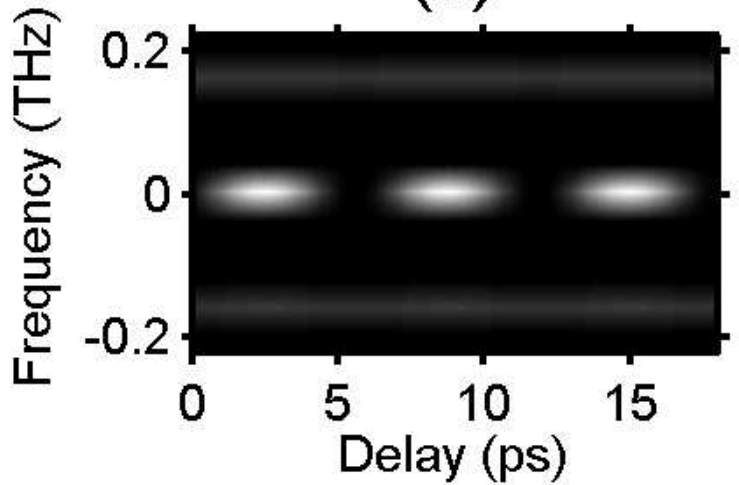

(d)

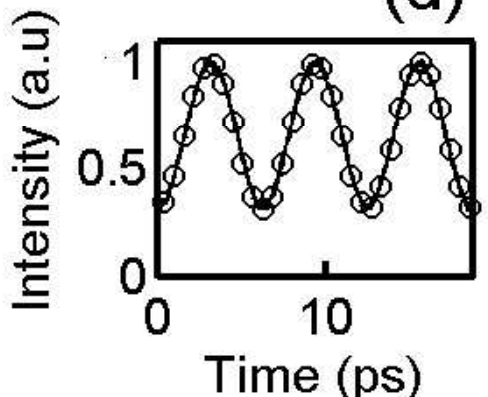

(e)

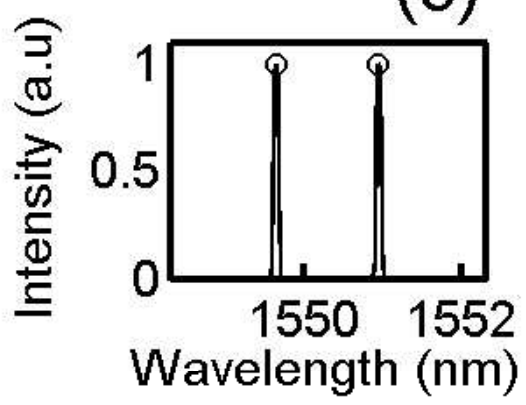


Fatome, Pitois, Millot

(a)

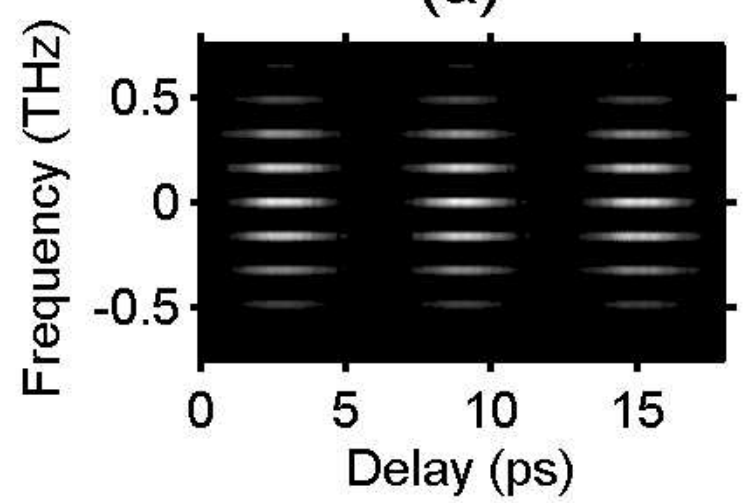

(c)

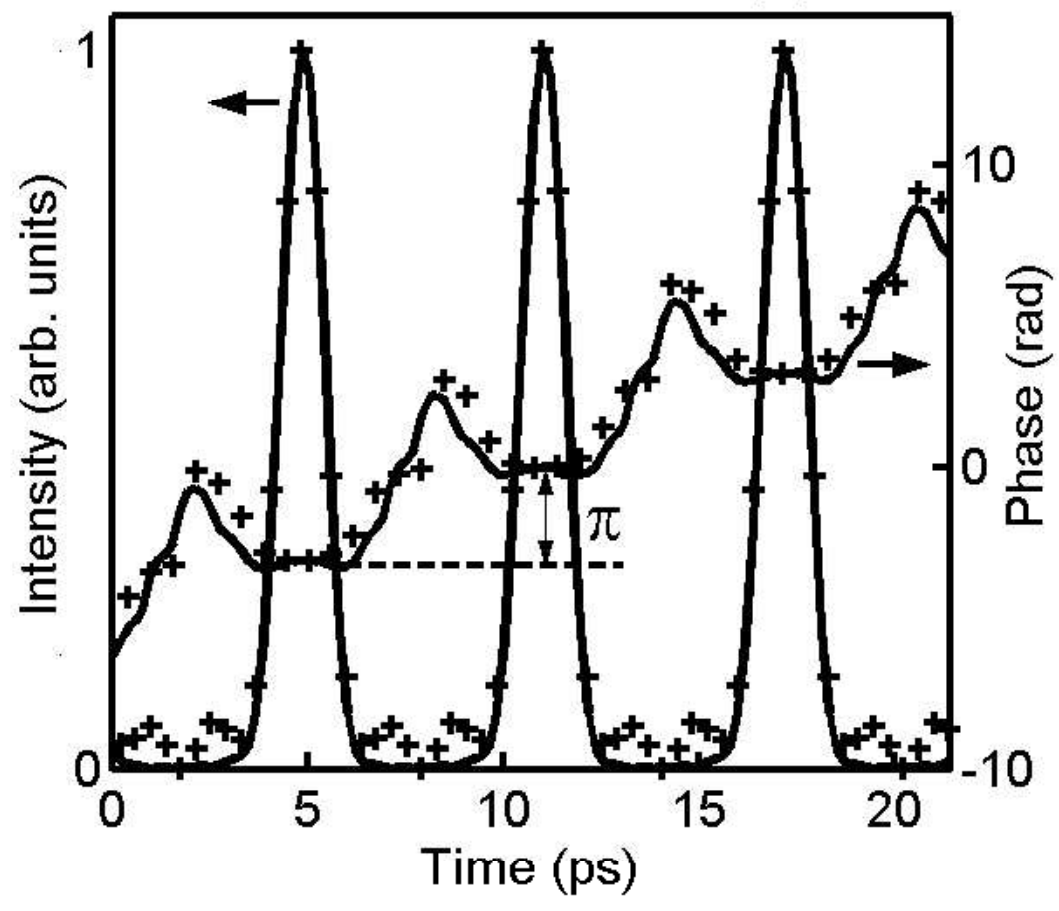

Fig. 3

(b)

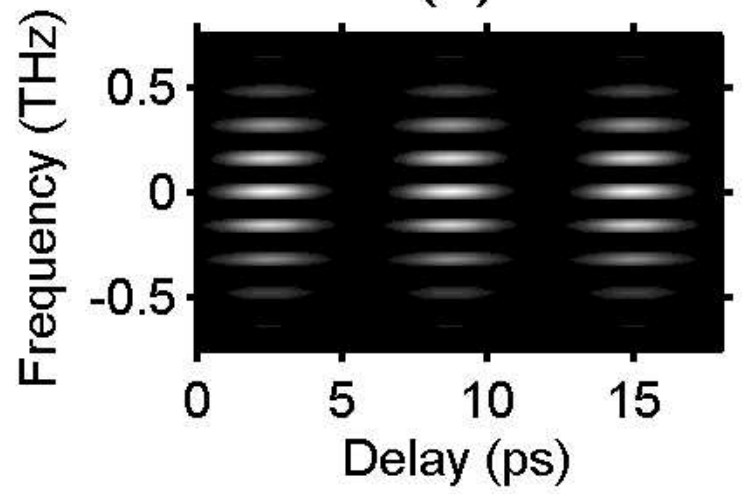

(d)

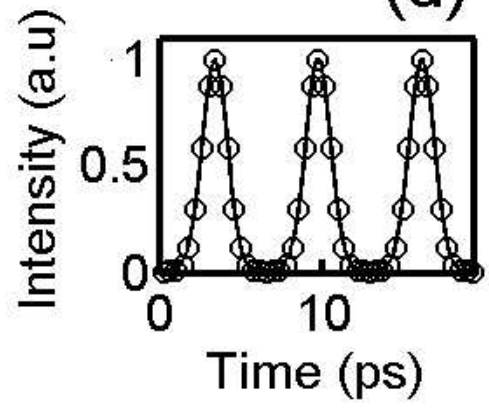

(e)

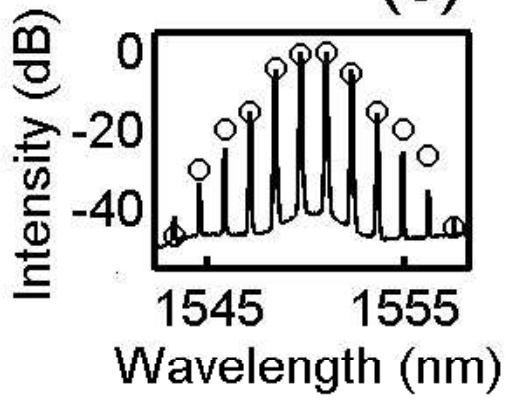

